\title{
SURGICAL MANAGEMENT OF METASTASIS OF PRIMARY VAGINAL CARCINOMA: ONE SINGLE INSTITUTE EXPERIENCE
}

\author{
M. Bouhani1, I. Zemni1, M.A. bouida1, M. slimene1, H. bouzaiene1, J. ben hassouna1, \\ M. hechiche1, R. chargui1, K. rahal1 \\ 1Salah Azaiz Institute, Oncologic Surgery, Tunis, Tunisia
}

Introduction:

Carcinoma of the vagina constitutes only about $2 \%$ of malignant neoplasm of the female genital tract. Because of the rarity of distant metastasis, there has been very limited data about managing primary vaginal cancer with distant metastasis.

\section{METHODS:}

We reported the surgical management of metastasis of primary vaginal in 2 patients treated in Salah Azaiez institute on 1994 and 2006.

\section{Results:}

\section{Case 1:}

We report a case of a 37-year-old woman who was diagnosed with a small cell carcinoma of the vagina without distant metastasis. The primary vaginal tumor was treated by surgery, brachytherapy, and chemotherapy. The first recurrence appeared seven years after initial diagnosis, which was a pulmonary metastasis, treated surgically. Seven years after this recurrence, she was treated with chemotherapy and surgery for another pulmonary metastasis. Fifteen years after the initial diagnosis, the patient underwent surgery for brain metastasis. She died eighteen months after the third recurrence.

\section{Case 2:}

We report a case of a 68-year-old woman who was diagnosed with adenocarcinoma of the vagina without distant metastasis. The tumor was classified as stage IV of FIGO.

The primary vaginal tumor was treated by hysterectomy with bilateral adnexectomy, and vaginectomy. In the surgical exploration, we discovered a lombo-aortique lymph node. The frozen section concluded to an inflammatory node, but the definitive exam showed a metastazing lymph node. Then the patient had an adjuvant radiotherapy and chemotherapy based on carboplatine combined with taxol for 4 cycles. One year later she had a local recurrence successfully treated with chemotherapy (carboplatine and taxol). Two years later, the patient had a cerebral metastasis from his vaginal tumor which was successfully removed. Than she had cerebral prophylactic radiotherapy; the patient was free of disease during her follow up period (2 years).

\begin{abstract}
Discussion:
Vaginal cancer is a rare entity composing only $1 \%$ to $2 \%$ of gynecologic malignancies. The role of surgery has been limited to initial stages. For early stage cancers, external beam radiotherapy or even brachytherapy alone have achieved excellent results in improving 5-year survival rate. Distant metastasis had been rarely reported in the literature, some studies showed a benefit of surgical resection in case of solitary metastasis. Others studies found a benefit in chemotherapy associated with radiotherapy. But optimal treatment is not yet established.
\end{abstract}

\section{Conclusion:}

We reported a successful surgical management of metastasis of primary vaginal carcinoma in two patients; our findings seem to be interesting due the lack of data about the management of those metastasis. 